\title{
Optokinetic backgrounds affect perceived velocity during ocular tracking
}

\author{
JANE E. RAYMOND and KIMRON L. SHAPIRO \\ University of Calgary, Calgary, Alberta, Canada \\ and \\ DEBRA J. ROSE \\ The Pennsylvania State University, University Park, Pennsylvania
}

\begin{abstract}
The perceived velocity of visually tracked moving objects may depend on interactions between reflexive and voluntary oculomotor mechanisms. To investigate this hypothesis, subjects were required to compare sequentially the velocity of a standard target with that of a test target moving at one of five velocities. The standard target was viewed against a plain field (no optokinetic stimulation), and the test target was viewed against (1) a plain field, (2) a stationary grating, (3) a grating drifting in the direction of target motion, or (4) a grating drifting against target motion. In one condition, subjects tracked the target; in the other, a stationary point was fixated. The tracking group overestimated velocity when backgrounds drifted against target motion, but underestimated velocity when gratings drifted with target motion. Subjects not required to track experienced no such misestimations. The results are discussed in relation to the interactive mechanisms of the two eye-movement systems.
\end{abstract}

The velocity of a moving object is perceived by different mechanisms, depending on whether the object's image is (1) moving relative to the retina, for example, with a stationary eye position, or (2) stationary on the retina and moving relative to the environment, as in ocular tracking. In the former condition, an object's velocity is signaled by neural units sensitive to movement of the image across the retina. However, if the eye is moving at exactly the same velocity as the object, as occurs in visual tracking, there is no movement of the retinal image and the perception of object motion is thought to result from an efference copy (von Holst \& Mittelstaedt, 1950), or corollary discharge (Teuber, 1960), of the eye movement. This suggests that the perception of velocity during visual tracking may also be derived from the efference copy signal. However, perceived stimulus velocity does not always correspond to the speed of the tracking eye movement. For example, the velocity of a moving grating pattern is underestimated during visual tracking (Dichgans, Wist, Diener, \& Brandt, 1975). This effect, known as the Aubert-Fleischl effect, has been explained by postulating that pursuit eye movements are slightly underregistered by the perceptual system (Mack \& Herman, 1973). This theory cannot predict velocity overestimation,

This research was supported by a postdoctoral fellowship to the first author from the Medical Research Council of Canada and by an NIMH grant (EYO32786) to Hersch Leibowitz. We thank Dr. Leibowitz for the use of laboratory facilities and both Dr. Leibowitz and Dr. Robert Dewar for their helpful comments on the preparation of this manuscript. This research was conducted in the Department of Psychology at The Pennsylvania State University. nor can it account for a variety of similar velocity perception phenomena related to eye movements during vestibular stimulation, for example, the oculogyral illusion (Whiteside, Graybiel, \& Niven, 1965). In this illusion, an observer and a fixated stimulus undergo equal, simultaneous acceleration. The fixated stimulus appears to move relative to the observer in the same direction as the acceleration.

A more parsimonious explanation for these distortions and illusions is based on the nature of the eye-movement systems used to track moving objects (Post \& Leibowitz, 1982; Whiteside et al., 1965). Visual tracking may be accomplished by either the voluntary pursuit system or by a reflexively controlled mechanism (Dichgans, 1977; Robinson, 1976). The voluntary system is typically used to track the image of small objects moving within the vicinity of the fovea. On the other hand, smooth reflexive eye movements, such as those seen during the slow phase of optokinetic nystagmus, are employed to maintain retinal image stability during head and body motion. Smooth reflexive eye movements can be elicited vestibularly by head motion or visually by movement of contours across large portions of the visual field. Although voluntary pursuit eye movements produce the sensation of object motion and are therefore thought to be accompanied by an efference copy, reflexively generated eye movements produce the sensation of self-motion and do not seem to generate the efference copy needed for object motion perception (Dichgans \& Brandt, 1976).

In most situations, the activity of the reflexive and voluntary smooth eye-movement mechanisms are probably combined. This interaction may be synergistic or an- 
tagonistic, depending on the stimulus conditions. Antagonistic interactions occur, for example, when a moving target is tracked against a stationary patterned field. In this situation, the voluntary pursuit eye movements used to track the object will produce movement of background contours across the retina in the opposite direction, thus eliciting an optokinetic response in the direction opposite to that required for object tracking. To maintain a stationary retinal image of the moving object, pursuit tracking must be performed while reflexive eye movements are suppressed. Since suppression is probably accomplished by additional activity of voluntary oculomotor mechanisms (Waespe \& Henn, 1977), tracking a moving object against opposite moving contours may place a greater demand on voluntary pursuit effort than tracking against a uniform field.

A synergistic interaction of voluntary and reflexive mechanisms may occur when contours in the visual background move in the same direction as a visually tracked moving object. In this case, reflexive tracking eye movements are elicited in the same direction as object motion and would reduce the effort required of voluntary tracking mechanisms. Support for both antagonistic and synergistic interactions between reflexive and voluntary eye-movement mechanisms has been reported recently by Yee, Daniels, Jones, Baloh, and Honrubia (1983). These investigators demonstrated that pursuit-tracking gain, that is, the ratio of eye velocity to stimulus velocity, was increased or decreased when a small moving target was tracked against an optokinetic background drifting in the same or opposite direction, respectively, as target motion, relative to tracking against an empty field. This suggests that optokinetically elicited eye movements interact with voluntary pursuit tracking.

If voluntary and reflexive eye-movement systems interact as suggested, then stimuli activating reflexive smooth eye-movement mechanisms would be expected to alter the effort required to voluntarily track a moving object. If the efference copy is determined by the effort required to track the moving object, rather than the speed or amplitude of the actual eye movement, then optokinetic stimulation would be expected to affect the efference copy and, hence, velocity perception. The present experiment investigated this possibility by assessing perceived velocity of a small moving object when it was visually tracked against backgrounds varying in their effectiveness as optokinetic stimuli. This set of experimental conditions was then compared with a second set of conditions, in which identical measures of perceived velocity were obtained with a stationary eye position.

\section{METHOD}

\section{Subjects}

Participants were eight female and six male student volunteers ranging in age from 18 to 31 years. All subjects had uncorrected or, where necessary, corrected, Snellen acuity of $20 / 30$ or better in both eyes, normal stereopsis, and normal lateral and vertical phorias, as measured with a Titmus Vision Tester prior to the experiment. The subjects were naive as to the purpose of the experiment and were inexperienced psychophysical observers.

\section{Apparatus}

Separate display monitors were used to generate the moving target and background stimuli. The moving target was displayed on a $23-\mathrm{cm}$ Sony monochrome monitor connected to a microcomputer (Apple Ile). Target motion was produced using high-resolution graphics software which successively illuminated two immediately adjacent pixels so that, as in phi movement, the target appeared to move smoothly across the screen. The interval between pixel on-time was varied to produce the necessary target speeds. Smooth pursuit tracking to phi motion, as used in the present experiment, is not different from that to real motion (Westhiemer, 1954). The speed of target motion was varied within the range required by the present experiment without changing the apparent size or brightness of the target.

The background stimuli were generated on a Tektronix (608) monitor (P31 phosphor) using analog signals from a Wavetek (193) function generator. Z-mod input to the monitor was switched by the microcomputer so that presentations of background stimuli were timed precisely.

The two monitors were positioned at right angles to one another. By placing a half-silvered mirror at a $45^{\circ}$ angle between them, the displays appeared to be superimposed to the subject. The subject monocularly (right eye) viewed the display from a distance of $35 \mathrm{~cm}$ through a field aperture $(1.3 \mathrm{~cm}$ in diameter) placed $4 \mathrm{~cm}$ from the eye. A chinrest was used to minimize head motion. The subject indicated his/her response by depressing one of two buttons.

\section{Stimuli}

A green moving target was viewed against one of four background displays. The moving target appeared as a horizontally oriented solid ellipse $0.25^{\circ}$ (visual angle) in length and $0.12^{\circ}$ in width with a luminance of $59.5 \mathrm{~cd} / \mathrm{m}^{2}$. The target traveled from left to right over a distance that randomly varied from $10^{\circ}$ to $16^{\circ}$ on successive presentations.

The background $\left(18^{\circ} \times 14^{\circ}\right)$ consisted of either a spatially uniform field or a 1-cycle/deg square-wave grating of $35 \%$ contrast. The grating was either stationary or drifting at a rate of $7.5 \mathrm{deg} / \mathrm{sec}$, either in the direction of target motion or against it. This created four different background conditions (plain, stationary, moving with, and moving against). In all cases, the mean background luminance was $13.2 \mathrm{~cd} / \mathrm{m}^{2}$.

The contrast between target and background was sufficiently high to permit clear detectability of the target at all times. In the stationary eye position condition, the subjects were provided with a fixation dot equal in brightness and size to that of the target. The fixation stimulus was located in the center of the screen $1^{\circ}$ below the path of the target.

\section{Procedure}

Each trail was composed of two target presentations. In the first presentation, a "standard" target appeared in the left half of the screen and moved horizontally toward the right at a constant velocity of $3.9 \mathrm{deg} / \mathrm{sec}$ across a spatially uniform field. The distance over which it traveled was one of five possible values $\left(10.0^{\circ}, 11.1^{\circ}, 12.5^{\circ}, 14.3^{\circ}\right.$, and $\left.16.0^{\circ}\right)$, presented in a random order. A tone signaled the end of the standard target presentation. After a 2-sec interval, the second, "test," target appeared on the left side of the screen and moved horizontally toward the right at one of five velocities against one of four background stimuli. The five different test velocities $(2.5,3.1,3.9,4.8$, and $6.5 \mathrm{deg} / \mathrm{sec})$ are separated by similar logarithmic intervals. As with the standard target, the excursion was one of the five possible values specified above in order that duration of stimulus presentation not be a reliable cue to target velocity. A different tone signaled the end of the test target presentation and cued the subject to respond by judging whether the test target had moved with the same or a different velocity as the preceding standard target. After responding by depressing one of two buttons marked "same" or "different," the next trial was initiated following a 3-sec interval. No feedback regarding performance was given at any time during the experiment.

Each experimental session consisted of four blocks of 50 trials each. Within each block, only one of the four backgrounds was presented. 
The order of blocks was varied randomly for each subject. Within each block, the excursion of both targets and the velocity of the test target were varied on each trial. The five different test velocities were presented in a pseudorandom order such that each velocity was presented 10 times per block of trials. The same five velocities were presented for all background conditions.

For half the subjects, no fixation spot was provided and subjects were told explicitly to visually track the moving target while maintaining a steady head position. The other half were instructed to maintain steady head and eye positions while fixating the dot provided.

Each experimental session began with a Titmus vision test followed by verbal instructions for the velocity perception task. Each subject was informed that target excursion would be an unreliable cue for judging target velocity. Ten practice trials in the presence of a single, randomly chosen background pattern were then given, and, after a brief interval, the experiment began. A rest period of approximately $5 \mathrm{~min}$ was provided between each block of trials. The experimental session lasted approximately $1 \mathrm{~h}$.

\section{RESULTS}

The present experiment demonstrates that the presence of optokinetic backgrounds affects the perceived velocity of a moving object during ocular tracking. This effect was not observed, however, when subjects were instructed to fixate a stationary point.

The percentage of trials on which subjects judged the test target to have the same velocity as the standard target was plotted as a function of log test velocity. The mean of this function reflects the test velocity perceived to match that of the standard target. Panel A of Figure 1 shows group mean responses obtained in the tracking condition for each background condition; Panel B shows the same relationship for the fixation condition.

An analysis of variance of the function means in the tracking condition revealed a significant effect of the back-

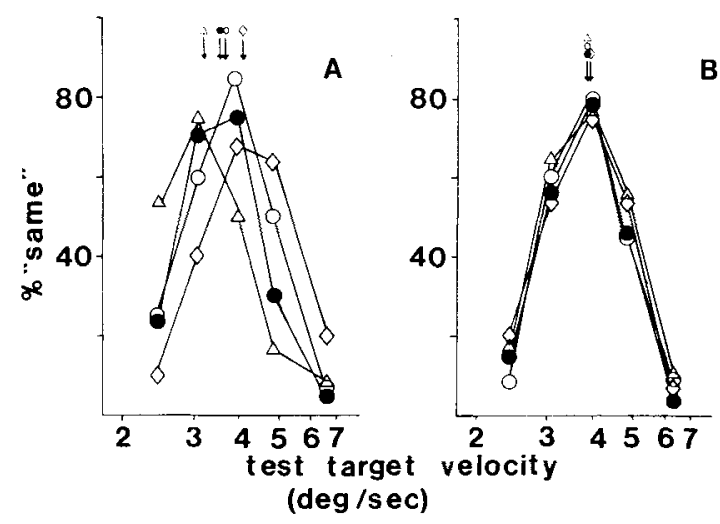

Figure 1. The group mean percentage of trials on which subjects judged the standard and test target velocity to be the same as a function of the test target's actual velocity. Panel A shows percent "same" responses obtained with visual tracking, whereas Panel B shows the same measure obtained in the fixation condition. Closed circles show the data obtained with a plain background; open circles indicate the stationary grating condition; open triangles indicate the condition with a grating drifting in the direction opposite to target motion; and open diamonds indicate the condition with a grating drifting in the same direction as target motion. The arrows at the top of the figure show the means of the corresponding functions. ground condition on perceived velocity $[F(3,24)=13.0$, $\mathrm{p}<.05]$. No significant effect of background was found in the steady fixation condition. With tracking, the test velocity chosen to match that of the standard was slower when the background drifted in the direction of target motion relative to that chosen in the plain (no background) condition $[t(24)=30, p<.05]$. The difference between the group mean values was $0.52 \mathrm{deg} / \mathrm{sec}$. On the other hand, when the background drifted against target motion, the group mean test velocity chosen was $0.32 \mathrm{deg} / \mathrm{sec}$ faster than that observed in the plain condition [t $(24=$ $20, p<.05]$. There was no difference between the group mean velocity obtained in the stationary and that obtained in the plain condition. An overestimation of velocity in the presence of the stationary background condition was expected. Sweeping the eye across this pattern during tracking should produce an optokinetic response. Since no velocity overestimation was observed in the present experiment, this suggests that the effectiveness of such stimulation may be limited.

\section{DISCUSSION}

The present experiment demonstrates that visual tracking of a moving object against a background of large moving contours produces a misperception of object velocity. Subjects over- or underestimated object velocity when background motion was in the opposite or same direction, respectively, as that of object motion. There was no effect in the tracking condition when background motion was not present, that is, in the plain and stationary backgrounds. Moreover, no alterations of perceived velocity were found when subjects viewed the various backgrounds but were instructed to fixate a stationary dot.

In the fixation condition, the relative motion of the background and target, or target and fixation point, may have provided an accurate cue for estimating target velocity under all background conditions. Under such circumstances, target velocity estimates are derived solely afferently from motion on the retina. No voluntary eye-movement activity is directed at the moving target, and thus no efference copy related to target motion is produced. With tracking, however, the image of the target remains stationary on the fovea while the retinal image of the background grating moves leftward or rightward, depending on the condition. Thus, there is no relative motion of target and background on the retina during tracking. Information regarding background motion is conveyed afferently from retinal motion detectors, whereas target movement is perceived via the efference copy produced by tracking eye movements. Clearly, afferent and efferent signals must interact to produce the velocity misperception effects observed in the present experiment. A mechanism for this interaction may be the synergistic and antagonistic activity of the voluntary and reflexive eye-movement systems.

Tracking eye movements in a complex visual environment probably represent the combined output of two 
oculomotor mechanisms: (1) the voluntary smooth pursuit system, and (2) the optokinetic (or vestibuloocular) reflexive smooth eye-movement system. While voluntarily produced eye movements seem to generate the efference copy necessary for motion perception during tracking, reflexively generated eye movements do not (Dichgans \& Brandt, 1976). Inaccurate velocity perception would thus be predicted when tracking eye movements are derived from both voluntary and reflexive eye-movement activity. Such would be the case whenever optokinetic stimuli were present. Support for this idea is found in the velocity misperceptions observed in the tracking condition of the present experiment in the presence of drifting optokinetic backgrounds.

The contribution of the reflexive system to total oculomotor movement during object tracking depends on the optokinetic effectiveness of the background. Background stimulus parameters, for example, field size, spatial frequency, and contour movement rate, have been shown to influence the gain of optokinetic nystagmus (Dichgans, 1977; Schor \& Narayan, 1981). In a tracking task, such parameters should therefore influence the ratio of reflexive to voluntary eye-movement activity, and hence the magnitude of the velocity misestimation. The magnitude of the effect observed in the present experiment is thus likely to be significantly less than that observed in the real world, given the large field size of naturally occurring optokinetic stimulation relative to the small field size of the background gratings used here.

It seems reasonable to expect that individuals can learn to compensate for velocity misperceptions such as those in the present study if provided with feedback. Since the vestibuloocular reflex is highly modifiable in healthy adults (Gonshor \& Melville-Jones, 1976), optokinetic eye movements also may be adjustable. Thus, misperceptions of velocity resulting from changes in visual stimulation may exist only until the magnitude of reflexive mechanisms is modified, or recalibrated, to suit the new perceptual situation. The implication is that acquisition of motor skills dependent on velocity perception may benefit from training in a variety of visual backgrounds, each of which differentially activates reflexive oculomotor responses. The feasibility of such an idea is evidenced by skilled marksmen, tennis players, and other athletes who can suc- cessfully perform velocity estimation tasks in the presence of a variety of visual and vestibular stimuli.

\section{REFERENCES}

DichGans, J. (1977). Optokinetic nystagmus as dependent on the retinal periphery via the vestibular nucleus. In R. Baker \& A. Berthoz (Eds.), Control of gaze by brain stem neurons. Amsterdam: NorthHolland/Elsevier.

DichGans, J., \& BRANDT, T. (1976). Visual-vestibular interactions: Effects on self-motion and postural control. In R. Held, H. W. Leibowitz, \& H. L. Teuber (Eds.), Handbook of sensory physiology (Vol. 8). Heidelberg: Springer.

Dichgans, J., Wist, E., Diener, H. C. , \& Brandi, T. (1975). The AubertFleischl phenomena: A temporal frequency effect on perceived velocity in afferent motion perception. Experimental Brain Research, 23, 529-533.

GonshOR, A., \& MELville-Jones, G. (1976). Short-term adaptive changes in the human vestibulo-ocular reflex. Journal of Physiology, 256, 361-379.

Mack, A., \& Herman, E. (1973). Position constancy during pursuit eye movements: An investigation of the Filehne illusion. Quarterly Journal of Experimental Psychology, 25, 71-84.

Post, R. B., \& LEIBowitZ, H. W. (1982). The effect of convergence on the vestibulo-ocular reflex and implications for perceived movements. Vision Research, 22, 461-467.

RoBinson, D. A. (1976). The physiology of pursuit eye movements. In R. A. Monty \& J. W. Senders (Eds.), Eye movements and psychological processes. Hillsdale, NJ: Erlbaum.

Schor, C., \& Narayan, V. (1981). The influence of field size upon the spatial frequency response of optokinetic nystagmus. Vision Research, 21, 985-994.

Teuber, H. L. (1960). Perception. In H. Magoun (Ed.), Handbook of physiology. III: Section on neurophysiology. Washington, DC: American Physiological Society.

von Holst, E., \& Mittelstaedt, H. (1950). Das reafferenz Prinzip, Wechselwirkungen zwischen Zentralnervensystem und Peripherie. Naturwissenschaften, 37, 464-476.

WAESPE, W., \& HENN, V. (1977). Vestibular nuclei activity during optokinetic alternystagmus (OKAN) in the alert monkey. Experimental Brain Research, 30, 323-330.

WESTHEIMER, G. (1954). Eye movement responses to a horizontally moving visual stimulus. A.M.A. Archives of Ophthalmology, 52, 932-941.

Whiteside, T. D. M., Graybiel, A., \& Niven, J. I. (1965). Visual illusions of movement. Brain, 88, 193-210.

YeE, R. D., Daniels, S. A., Jones, O. W., Baloh, R. W., \& HonRUBIA, V. (1983). Effects of an optokinetic background on pursuit eye movements. Investigative Ophthalmology and Visual Science, 24, $1115-1122$.

(Manuscript received March 15, 1984; revision accepted for publication August 1, 1984.) 